\title{
LA-UR-20-27978
}

Approved for public release; distribution is unlimited.

Title: $\quad$ Spot size and pulse-to-pulse beam motion

Author(s): $\quad$ Schulze, Martin E.

Intended for: $\quad$ Report

Issued: 
Disclaimer:

Los Alamos National Laboratory, an affirmative action/equal opportunity employer, is operated by Triad National Security, LLC for the National Nuclear Security Administration of U.S. Department of Energy under contract 89233218CNA000001. By approving this article, the publisher recognizes that the U.S. Government retains nonexclusive, royalty-free license to publish or reproduce the published form of this contribution, or to allow others to do so, for U.S. Government purposes. Los Alamos National Laboratory requests that the publisher identify this article as work performed under the auspices of the U.S. Department of Energy. Los Alamos National Laboratory strongly supports academic freedom and a researcher's right to publish; as an institution, however, the Laboratory does not endorse the viewpoint of a publication or guarantee its technical correctness. 
M. Schulze

14-March-2011

Rev 1: 1-October-2020

\section{Spot size and pulse-to-pulse beam motion}

\section{Overview:}

Measurements made on March 1, 2011 show that the measured spot sizes are sensitive to the relative beam motion between the four kicked pulses. This note presents an analysis of this data and concludes that large relative beam motion between $\mathrm{P} 1$ and the succeeding pulses can cause the later pulses to become larger and/or distorted. The magnitude of the spot size increase is dependent upon the spacing between the pulses as the beam approaches the target. The measured spot sizes are significantly distorted if the pulse-to-pulse spacing is large relative to the spot size. For multi-pulse targets, the trajectories and shape of each pulse are not the same. This note presents evidence that the plume of the vaporized target material from prior pulses can cause a shift in the subsequent pulses towards the hole in the target from prior pulses thereby distorting the pulse.

\section{Analysis:}

The beam position on target is measured by the GRC camera $(.049 \mathrm{~mm} / \mathrm{pixel})$. We can al so calculate the beam position on target from the trajectory defined by BPM29 and BPM30. The two BPM's are upstream of the final focus magnet separated by a drift space. This trajectory is then propagated through the final focus magnet to determine the beam position on target. In order to accommodate misalignments inherent in the BPMs, the GRC beam positions are then shifted so that the $P 1$ position is the same as that calculated from BPM29 and BPM30. Shots 13765, 13766 and 13767 are compared. The only difference between these three shots is the vertical steering and vertical spacing between the four pulses. These shots were for the 3652 pulse format (four short pulses of 20-25 nsec). These shots are compared to shot 13085 which was the tune for 3652 . Note the following differences between the three shots on 3-1-11 and 13085:

1. A tuning $V$ was performed for 13085 but not prior to 3-1-11

2. Cell 26 was off for 13085 and on for 3-1-11

3. The final focus setting was $478 \mathrm{~A}$ for 13085 and $382 \mathrm{~A}$ for 3-1-11 corresponding to the energy difference with cell 26 off.

4. Cell solenoid 73 resistance was reduced to $84 \%$ on 3-1-11 compared to 9-24-10 and compensated by increasing the current by the reciprocal of the resistance change.

These differences will not significantly affect the spot size but will affect the relative beam motion. Figure 1 compares the measured (GRC) and calculated beam positions on target for these four shots. The $\mathrm{P} 1$ position is at the far left for all four shots and the P2, P3 and P4 are each successively further from the $P 1$ position in all cases for both the measured GRC and calculated positions. The measured beam positions for P2, P3 and P4 are shifted closer to P1 compared to the calculated position in all cases. Table 1 presents the measured shifts in the P2, P3 and P4 positions for these shots. The shifts are largest in the horizontal plane which corresponds to the vertical plane upstream of the final focus 
magnet. The magnitude of the shifts are largest when the difference between the measured and calculated beam positions are largest.

Figure 2 is the same as Figure 1 with the addition of an ellipse representing the measured spot size. Here the "smoothed" ellipse represents a one sigma semi-major and semi-minor radii based on the measured spot size. The measured spot sizes for the four shots are given in Table 2 . It is clear that the horizontal beam shift is correlated with an increased horizontal beam size and ellipticity. Considering that the only change in shots 13675 to 13677 is the vertical steering, it is clear that the elliptical shape of the beam spot in the later pulses is caused by the interaction of the pulse with the target environment resulting from prior pulses.

Figures 3-6 present the actual $x$-ray images in false color for these four shots. The P3 and P4 images for 13765 and 13766 show significant halo with the peak of the distribution shifted significantly in the horizontal plane as suggested by the above discussion. The P3 and P4 images for 13767 also appear to be slightly shifted which could explain the somewhat larger horizontal widths. The central "hot" spot for the later pulses in shots 13765 and 13766 is shifted causing long tails in the distribution. The difference between the measured and calculated beam positions is smallest for shot 13085 and the beam sizes and shapes are the smallest and roundest for this shot.

\section{Discussion/Conclusion:}

This note demonstrates the importance of minimizing beam motion on target and highlights the importance of performing a tuning $\mathrm{V}$ prior to any multi-pulse target studies. The tuning $\mathrm{V}$ performed on 9-8-10 achieved results that were better than the baseline requirements. At this time, a strong argument for tightening the baseline requirement for beam motion at the accelerator exit cannot be made.

The beam motion as measured at the target can be much smaller than the beam motion calculated on the basis of the beam trajectory as described above. The trajectories of the later pulses appear to be shifted due to the interaction of the beam with the vaporized target material from prior pulses. The above analysis shows that for later pulses that are significantly offset from the prior pulses, the beam will be shifted towards the location of the prior pulses. The challenge is to quantify the amount of offset that can be tolerated and not result in an increased spot size or distorted beam spot.

The present beam motion requirements were based on the argument that the beam motion should be small relative the spot size on target. It has never been established whether this should beam radius, beam diameter or FWHM. A useful measure of the offset is the radius of the circle that contains the centroid of all four spots. In practice, efforts are made to minimize this radius with a goal of keeping the radius below 250 um. Figure 7 shows this for the four shots considered above for both the measured spot size on target and the calculated location using BPM29 and BPM30. From Figure 7 it is clear that the measured beam centroids are in close agreement with the calculated beam centroids and the two radii are close for shot 18085 . This is not the case for the three other shots in which the radii of the measured beam centroids are smaller than the calculated radii. This can be deceiving for spots that are asymmetric or distorted as seen for shot 13765 in which the measured centroid radius is just over the goal of 250 um at 295 um while the P4 beam size is unacceptably large. In this case, the P4 beam shape would help to identify the problem. 
The sensitivity of the beam spots to the relative beam motion of the four pulses described above is one specific case. In some cases, the measured beam motion exceeds the goal of $250 \mathrm{um}$ and there is little evidence of distorted beam spots while in other cases there are clear indications that the relative beam motion does appear to contribute to the spot size. In general, it has been observed that the relative spacing shows $\mathrm{P} 2, \mathrm{P} 3$ and $\mathrm{P} 4$ each being successively further from $\mathrm{P} 1$ and occasionally $\mathrm{P} 3$ is closer to P1 than P2. Figure 8 shows an example of beam centroid motion that exceeds the nominal requirement of 250 um in which the measured spot sizes were very good and $P 2$ and $P 3$ are approximately equidistant from $\mathrm{P} 1$. The beam centroid motion is dominated by the $\mathrm{P} 1$ and $\mathrm{P} 4$ centroids for this and most all shots. It is possible that the relative positions of P1-P4 are related to the energy because each successive pulse typically has a higher energy than the earlier pulses. This feature coupled with the large horizontal steering at the accelerator exit could be a cause of this beam motion and needs furtherstudy.

To this end, it would be beneficial to investigate the limitations of the tuning V. Additional improvements could be realized by improving the cell voltage flattop and reducing the large horizontal steering that to date results from the tuning V. Another approach that has been discussed is to tune the accelerator so that the beam motion in the kicker (position and angle) is minimized in the horizontal plane and then to tune the kickervol tage for each pulse to further reduce the beam motion on target. This approach coupled with meeting the beam motion requirements at the accelerator exit could significantly reduce the beam motion on target. New approaches to reduce the steering at the accelerator exit and improve the beam motion on target are needed.
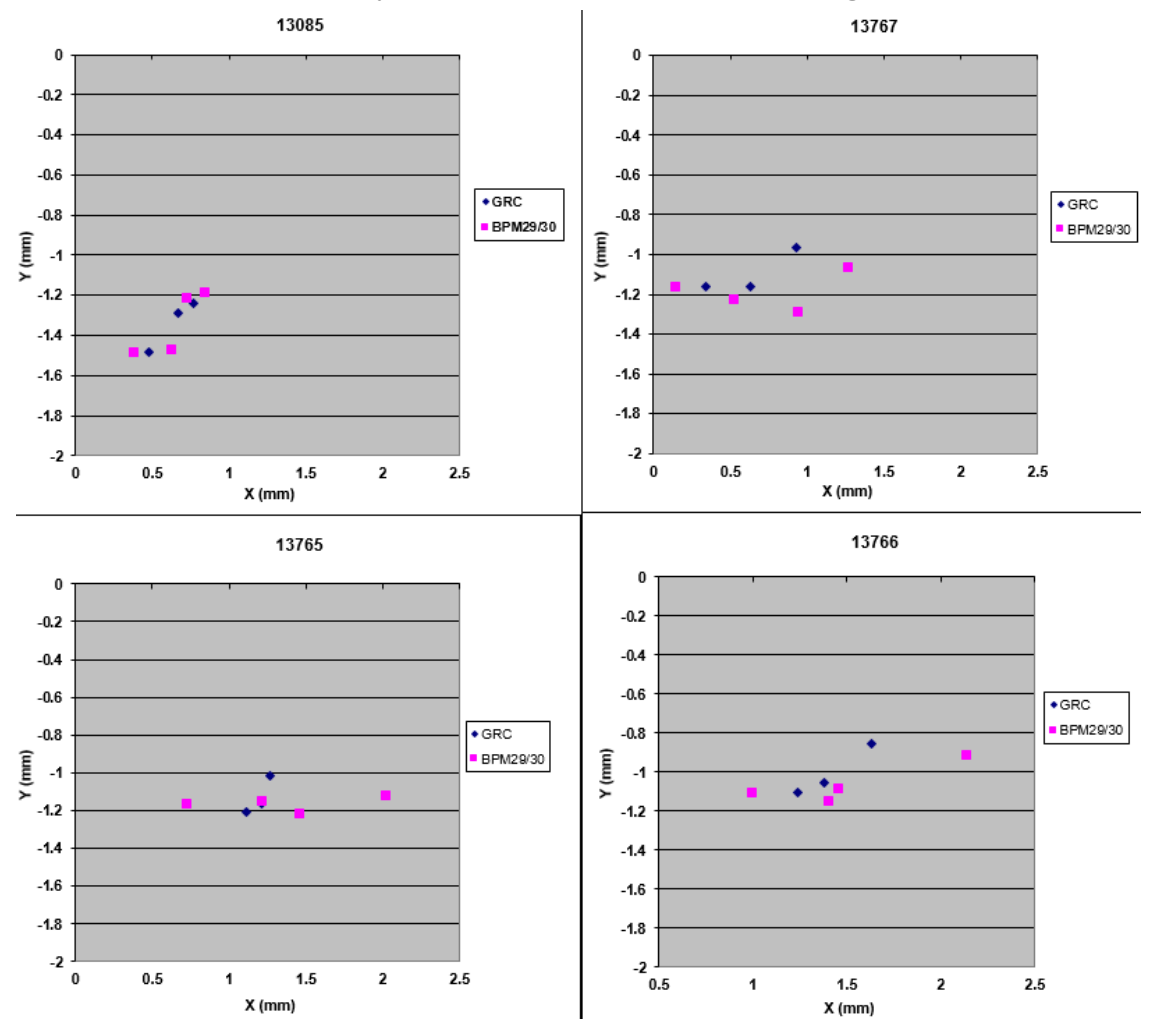

Figure 1: Measured and calculated beam position on target for shots 13085, 13767, 13766 and 13765 clockwise from top left. The GRC position is shifted so that it is in the same location as the calculated P1 position. 
Table 1: Shift in beam position between measured and calculated beam positions

\begin{tabular}{|l|r|r|r|r|r|r|r|r|}
\cline { 2 - 9 } \multicolumn{1}{c|}{} & \multicolumn{2}{c|}{13085} & \multicolumn{2}{c|}{13767} & \multicolumn{2}{c|}{13766} & \multicolumn{2}{c|}{13765} \\
\cline { 2 - 9 } \multicolumn{1}{c|}{} & \multicolumn{1}{c|}{$\mathrm{X}(\mathrm{mm})$} & \multicolumn{1}{c|}{$\mathrm{Y}(\mathrm{mm})$} & \multicolumn{1}{c|}{$\Delta \mathrm{X}(\mathrm{mm})$} & \multicolumn{1}{c|}{$\mathrm{Y}(\mathrm{mm})$} & $\Delta \mathrm{X}(\mathrm{mm})$ & $\Delta \mathrm{Y}(\mathrm{mm})$ & $\Delta \mathrm{X}(\mathrm{mm})$ & $\Delta \mathrm{Y}(\mathrm{mm})$ \\
\hline P2 & 0.1483 & 0.012 & 0.1794 & -0.0596 & 0.0931 & 0.0595 & 0.1627 & -0.0447 \\
\hline P3 & 0.0543 & 0.0731 & 0.3003 & -0.125 & 0.2388 & -0.0548 & 0.0679 & -0.0265 \\
\hline P4 & 0.0716 & 0.0493 & 0.337 & -0.0976 & 0.7537 & -0.1053 & 0.51 & -0.0573 \\
\hline
\end{tabular}

Table 2: Measured spot sizes for 13085, 13767, 13766 and 13765

\begin{tabular}{|r|r|r|r|r|r|r|}
\cline { 2 - 7 } \multicolumn{1}{c|}{} & \multicolumn{3}{c|}{ P1 } & \multicolumn{3}{c|}{ P2 } \\
\cline { 2 - 7 } & $X_{\text {FWHM }(\mathrm{mm})} \mathrm{Y}_{\text {FWHM }}(\mathrm{mm})$ & $50 \% \mathrm{MTF}(\mathrm{mm})$ & $\mathrm{X}_{\text {FWHM }}(\mathrm{mm})$ & $\mathrm{Y}_{\text {FWHM }}(\mathrm{mm})$ & $50 \% \mathrm{MTF}(\mathrm{mm})$ \\
\hline 13085 & 0.59 & 0.748 & 1.41 & 0.62 & 0.7 & 1.39 \\
\hline 13767 & 0.605 & 0.582 & 1.213 & 0.769 & 0.622 & 1.437 \\
\hline 13766 & 0.725 & 0.616 & 1.356 & 0.97 & 0.664 & 1.713 \\
\hline 13765 & 0.743 & 0.628 & 1.422 & 0.883 & 0.64 & 1.575 \\
\hline & \multicolumn{3}{|c|}{$\mathrm{P} 3$} & \multicolumn{3}{c|}{ P4 } \\
\cline { 2 - 8 } & $\mathrm{X}_{\text {FWHM }(\mathrm{mm})} \mathrm{Y}_{\text {FWHM }}(\mathrm{mm})$ & $50 \% \mathrm{MTF}(\mathrm{mm})$ & $\mathrm{X}_{\text {FWHM }}(\mathrm{mm})$ & $\mathrm{Y}_{\text {FWHM }}(\mathrm{mm})$ & $50 \% \mathrm{MTF}(\mathrm{mm})$ \\
\hline 13085 & 0.81 & 0.93 & 1.79 & 0.96 & 0.96 & 1.92 \\
\hline 13767 & 1.108 & 0.774 & 2 & 0.972 & 0.843 & 1.861 \\
\hline 13766 & 1.341 & 0.941 & 2.515 & 1.744 & 1.083 & 3.16 \\
\hline 13765 & 1.275 & 0.912 & 2.416 & 2.205 & 1.314 & 3.92 \\
\hline
\end{tabular}
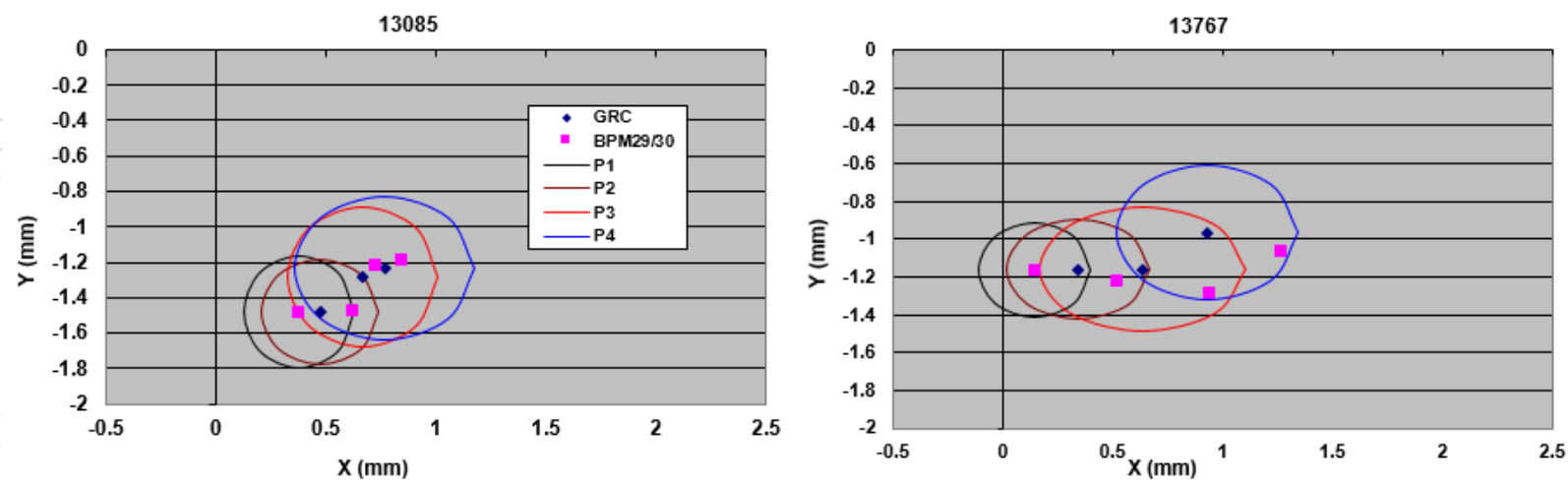

13765
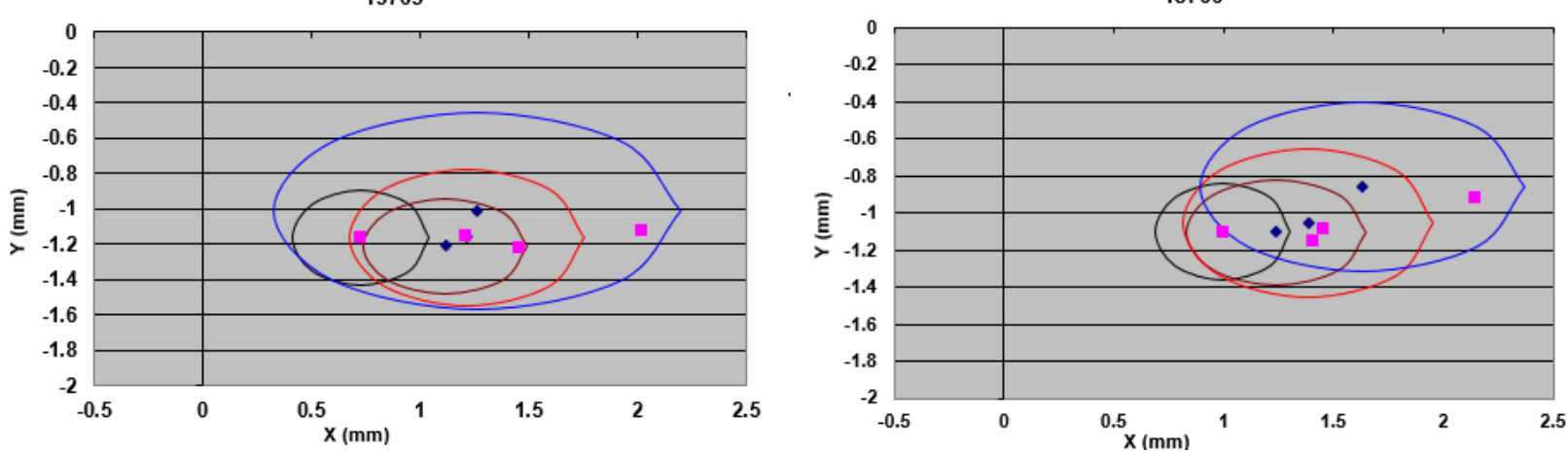

Figure 2: Measured and calculated beam position on target for shots 13085, 13767, 13766 and 13765 clockwise from top left. The GRC position is shifted so that it is in the same location as the calculated P1 position. The measured spots sizes are depicted by ellipses centered on the measured GRC beam position. 


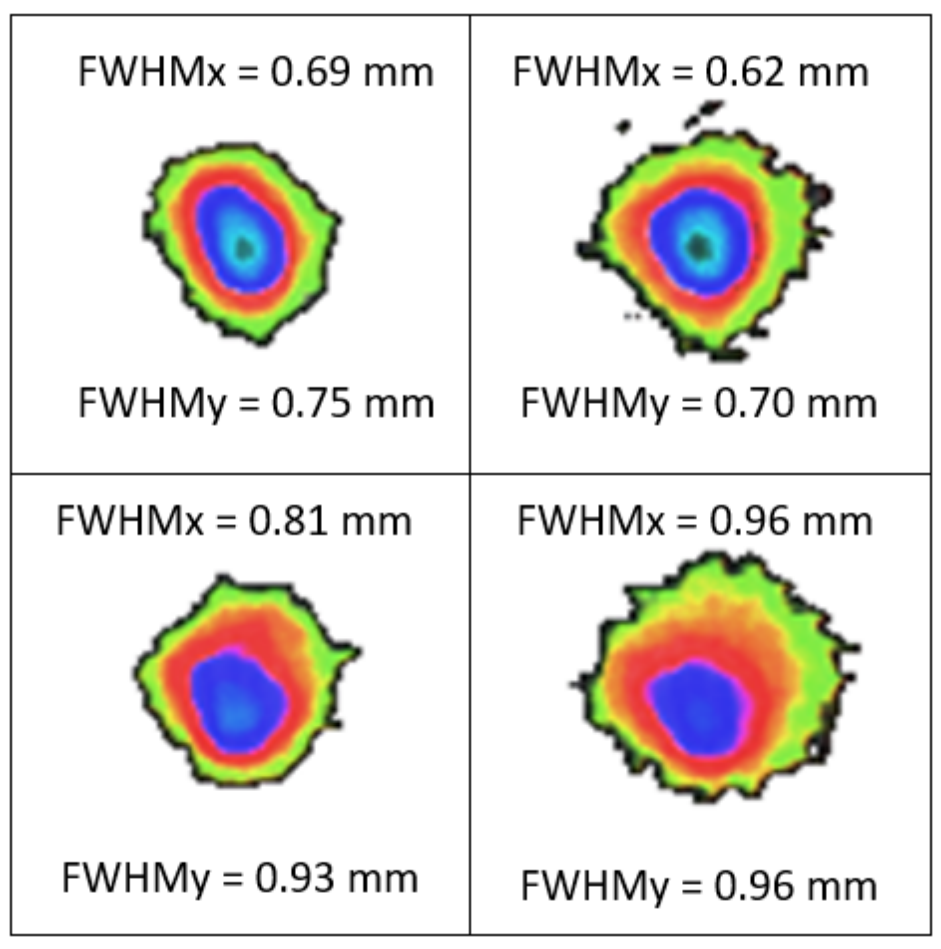

Figure 3: 13085 x-ray images with P1 in upper left, P2 in upper right, P3 in lower left and P4 in lower right.

\begin{tabular}{|l|l|}
\hline FWHMx $=0.61 \mathrm{~mm}$ & FWHMx $=0.77 \mathrm{~mm}$ \\
\hline FWHMy $=0.58 \mathrm{~mm}$ & FWHMy $=0.62 \mathrm{~mm}$ \\
\hline FWHMx $=1.11 \mathrm{~mm}$ & FWHMx $=0.97 \mathrm{~mm}$ \\
\hline FWHMy $=0.77 \mathrm{~mm}$ & FWHMy $=0.84 \mathrm{~mm}$ \\
\hline
\end{tabular}

Figure 4: 13767 x-ray images with P1 in upper left, P2 in upper right, P3 in lower left and P4 in lower right. 


\begin{tabular}{|l|l|}
\hline FWHMx $=0.73 \mathrm{~mm}$ & FWHMx $=0.97 \mathrm{~mm}$ \\
\hline FWHMy $=0.62 \mathrm{~mm}$ & FWHMy $=0.66 \mathrm{~mm}$ \\
\hline FWHMx $=1.34 \mathrm{~mm}$ & FWHMx $=1.74 \mathrm{~mm}$ \\
\hline
\end{tabular}

Figure 5: 13766 x-ray images with P1 in upper left, P2 in upper right, P3 in lower left and P4 in lower right.

\begin{tabular}{|l|l|l|}
\hline FWHMx $=0.74 \mathrm{~mm}$ \\
\hline FWHMy $=0.63 \mathrm{~mm}$
\end{tabular}

Figure 6: 13765 x-ray images with P1 in upper left, P2 in upper right, P3 in lower left and P4 in lower right. 

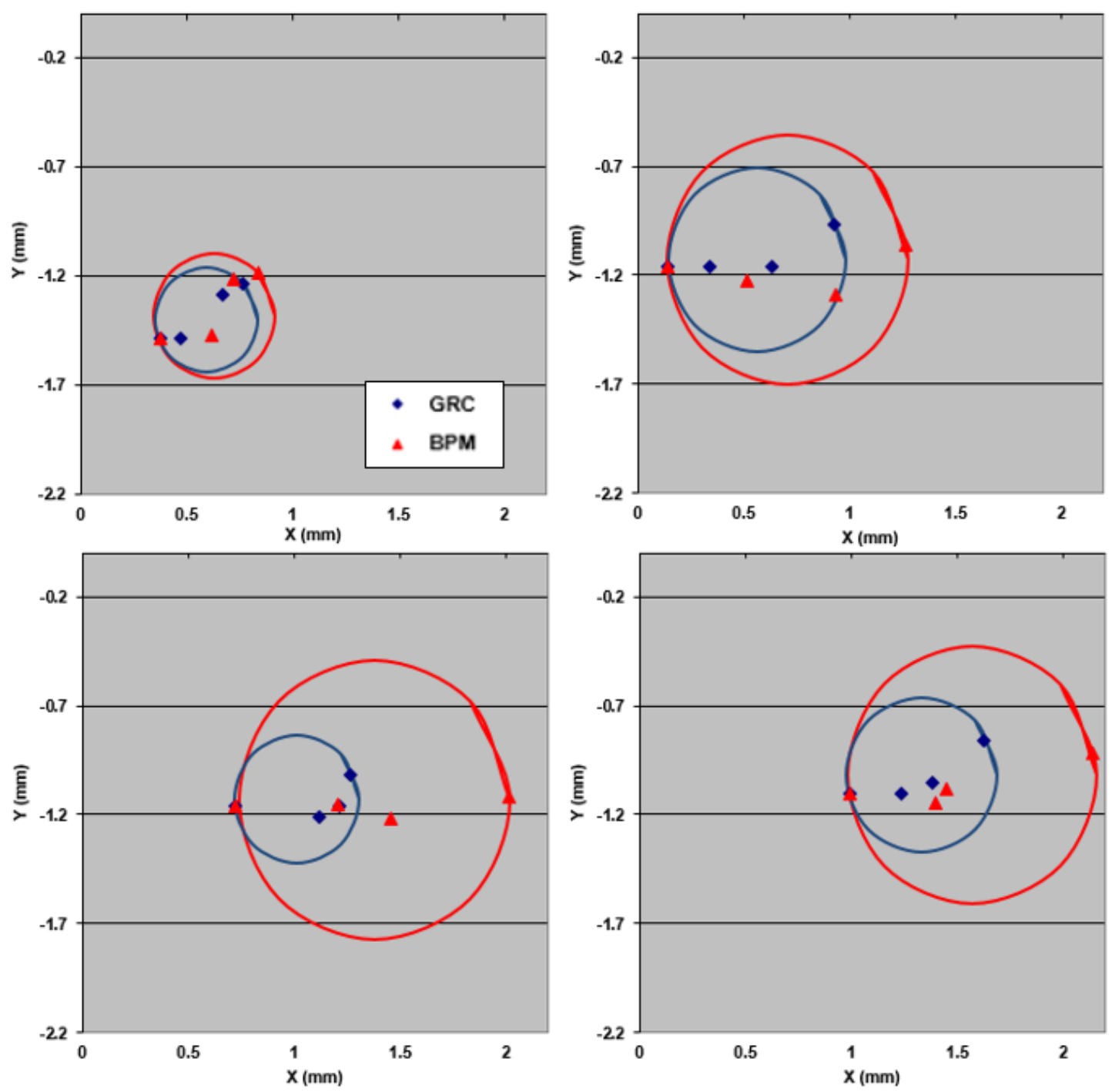

Figure 7: These four figures show the circle that inscribes the centroid of the four pulses for shots 13085 (upper left), 13767 (upper right), 13766 (lower right) and 13765 (lower left). The blue diamonds and circle are for the measured GRC spot. The red triangle and circle are for the calculated spot on target using BPM29 and BPM30. The horizontal and vertical scales are the same for all four figures.

Table 3: Radii of the beam centroids for the measured GRC and calculated BPM spots for shots 13085 , 13767, 13766 and 13765.

\begin{tabular}{|l|r|r|r|r|}
\cline { 2 - 5 } \multicolumn{1}{c|}{} & \multicolumn{4}{c|}{ Shot } \\
\cline { 2 - 5 } \multicolumn{1}{c|}{} & 13085 & 13767 & 13766 & 13765 \\
\hline GRC spot radius $(\mathrm{mm})$ & 0.24 & 0.42 & 0.355 & 0.295 \\
\hline BPM spot radius $(\mathrm{mm})$ & 0.285 & 0.57 & 0.59 & 0.64 \\
\hline
\end{tabular}




\section{Spot Position}

Radus $\{(\Delta m): 357$

Center: 256.0, 258.5

$$
\begin{aligned}
& P 1=X \\
& P 2=\text { square } \\
& P 3=\text { triangle } \\
& P 4=\text { diamond }
\end{aligned}
$$

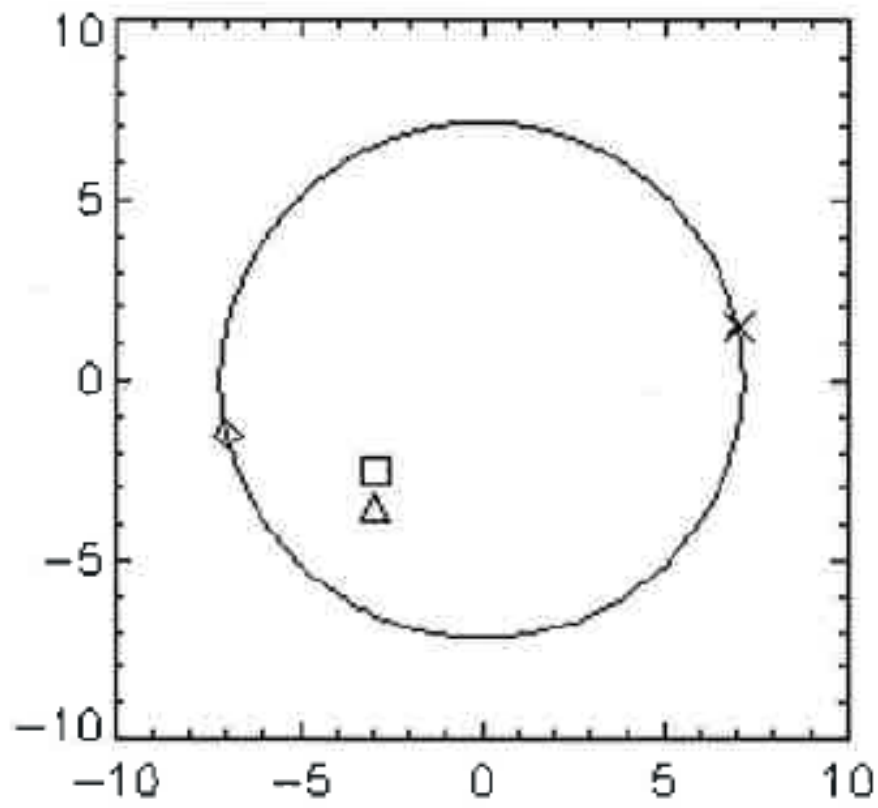

Figure 8: Beam centroid motion on target for shot 35637 taken in preparation for 4019 taken on April 18,2019 . The beam centroids are plotted in pixels $(.049 \mathrm{~mm} /$ pixel) relative to the center on the circle inscribing the four beam centroids. 